\title{
Review of Work Embedding Theory
}

\author{
Xie Yating ${ }^{1, a}{ }^{*}$ \\ ${ }^{1}$ School of Business Administration,Zhejiang GongShang University,Hangzhou,Zhejiang,China \\ a*2278200600@qq.com
}

\begin{abstract}
Work embedding theory has developed rapidly in recent years and plays an important role in predicting employee dimission.Based on the literature of work embedding at home and abroad in recent years, this paper clarified the concept of work embedding theory, introduced the methods of literature measurement in detail, summarized and sorted out the antegenics and outcome variables of work embedding, explored other relatively innovative research directions, and put forward research prospects on the basis of this literature review.
\end{abstract}

Keywords: employees, work embedding, employee turnover, lowercase letters

\section{工作嵌入理论研究综述}

\author{
谢亚婷 ${ }^{1, a}{ }^{*}$
}

${ }^{1}$ 浙江工商大学工商管理学院, 杭州, 浙江, 中国

${ }^{a *} 2278200600 @ q q . c o m$

\begin{abstract}
摘要
工作嵌入理论近几年有了很快的发展，在预测员工离职等员工行为方面有着十分重要的作用。本文基 于近几年中外工作嵌入的文献, 厘清嵌入理论概念, 对文献测量的方法进行详细介绍, 总结和梳理了 工作嵌入的前因和结果变量, 发掘了其他比较创新的研究方向, 并在此文献综述的基础上提出研究展 望。
\end{abstract}

关键词：员工；工作嵌入；员工离职；会议

\section{1. 引言}

随着市场经济的发展, 企业对人才越来越重视, 员 工离职问题一直是企业的关注重点。从1969年开始到现 在, 员工离职问题的话题一直方兴未艾, 员工的自动离 职给组织带来人力资本的损失以及招聘培训成本增加等 方面损失（Peterson \& Luthans, 2006）。1995年Tom Lee 和他的同事进行的员工离职的研究, 他们从员工为什么 离开的问题转移到人们为什么留下的问题, 研究团队开 始关注员工留下的原因, 那时工作嵌入的概念就此产生 (William Lee等, 2014)。研究者致力于去探索员工留 在组织的因素。现有的研究把员工嵌入与员工离职率、 工作绩效、创新绩效、工作满意度等因素进行相关分析, 工作嵌入也是预测员工工作绩效等工作表现的重要的变 量。

以往也有学者对于工作嵌入文献综述, 我国学者杨 春江在2010年发文关于工作嵌入的文献综述, 厘清了工 作嵌入的概念结构并对 “展开” 模型进行详尽的介绍 ${ }^{[1]}$, Mian Zhang等 (2012) 在2012年发布文章, 从工作嵌入概
念、测量和变量效度等方面进行全面的论述。在 2013

年, Thomas William Lee等 (2013) 以工作嵌入拓展的 前因和变量作为研究重点, 对于工作嵌入的衍生研究 方向进行了很好的梳理。从2013年至今文献综述类的 文章比较少, 没有对现在工作嵌入的发展进行一个完 整的梳理。在此基础上, 本文在将从以下几个方向对 于文献进行梳理:

首先是对工作嵌入的内涵进行阐述, 厘清的三个 指标的概念和含义。其次, 对于工作嵌入的衡量指标 进行论述, 然后, 分析整理与员工嵌入相关的相关的 前因、结果变量以及几年的研究拓展方向的梳理。最 后, 对于以上的分析进行总结和归纳, 提出未来的研 究展望。本文希望对于工作嵌入的研究有更好的梳理, 能为工作嵌入方向研究的人员提供一些参考。

\section{2. 工作嵌入的内涵}

工作嵌入性被认为是把员工留下来的力量, 让员 工深深的陷入到工作环境和社区环境之中，它被定义 为是对于员工财务、心理等方面产生影响，而使员工 


\section{4. 员工嵌入的研究现状及相关影响因素}

研究员工做出离开或者选择留下来的因素都是从组织环 境出发, 但是工作嵌入度的研究把对于员工生活环境和 社区的因素也包括进来, 提出员工也会因为对于对于生 活环境的偏爱也选择留下来。人生活的工作环境和生活 环境都好比是一个运转的相互影响的点的集合, 工作嵌 入度越高, 则是代表员工陷入这个集合的程度越深, 从 而影响员工选择留下来的决策。研究学者提出工作嵌入 是包括链接、匹配和牺牲三个指标, Lee等人为了区分开 在组织层次和社区层次不同的嵌入, 提出两类指标, 一 类是职业内嵌入, 一类是职业外嵌入。第一类指的是关 于组织层面的影响员工嵌入度的因素, 第二类指的是在 社区层面上对员工产生影响的要素。

链接被定义为一个组织和社区中, 个人和其他人之 间的正式的和非正式的联系 (Lee 等, 2004;Mitchell等, 2001） ${ }^{[3]}$ 。匹配被定义为个人与组织和社区之间的契合度 (Lee 等, 2004;Mitchell等, 2001)。当个人所需求的与 组织和社区能提供的可以相契合, 则可以说明人与组织 和社区是相容的。在组织和社区中匹配度高的情况下, 个人会更愿意留在当前的组织和社区中。牺牲是个体离 开当前的组织和社区所感觉到心理上和经济上所付出的 成本的集合（Zhang, M.等，2012）。

\section{3. 工作嵌入测量}

目前是主要是有三种工作嵌入性的测量方式, Mitchell和Lee（2001）提出的工作嵌入的测量方法 ${ }^{[4]}$, Crossley等 (2007) 提出的全球工作测量度的测量方法 ${ }^{[5]}$, Clinton等 (2012) 人提出的双尺度测量量表 ${ }^{[6]}$, 还有学者 积极在开发出不同文化背景下的员工嵌入的量表, 如我 国学者杨春江 (2019) 开发出中国文化背景下的测量量 表, 四种的测量量表在指标、方法和效度上有所不同 ${ }^{[7]}$ 。

Mitchell和Lee (2001) 是最先提出工作嵌入度量表的 学者, 其开发的量表由六个维度形成的聚合多维结构, 工作嵌入不是一个统一的结构一它是工作上和工作外 的多维聚合 (Mitchell等, 2001)。Crossley等 (2007) 在 2007年提出的全球工作测量度的测量方法, 采用的是7个 题项的题项测量方式, 通过7个题项对于工作嵌入问题进 行整体的测量。该测量的信效度较好, 该方法可以在整 体上测量参与者对于组织的依恋程度。Clinton等 (2012) 在此基础上提出的双因素测量量表。他使用双因素的概 念, 既可以用更简短的问题测量, 又能兼顾组织和社区 两个层面。达到用简单因素的测量出工作嵌入的整体构 念。杨春江考虑到在不同文化背景下员工嵌入的侧重有 所不同, 所以致力于开发中国文化下的测量方式, 该研 究是在借鉴Mitchell等人研究, 结合中国情景, 把嵌入分 为组织和区域的两个范畴和三种关系类型下的多维结 构。
自员工嵌入提出来后, 研究学者对于该变量进行 多方面的研究, 员工嵌入独立于其他的变量, 作为预 测员工行为的一个重要的部分, 员工嵌入理论也被广 泛的应用。本部门对于员工嵌入影响的前因变量和后 效变量进行探讨, 以及总结近几年研究的拓展。

\section{1. 前因变量研究综述}

关于前因变量的研究, 研究学者主要是从组织和 社区角度去发现对于员工嵌入有促进的因素。Barjinder Singh等（2018）基于资源保护理论（COE）来开发组 织和社区嵌入相关的环境和个人资源模型, 提出支持 的角色、心理安全和归属的需要这三个变量对于促进 员工嵌入的重要作用 ${ }^{[8]}$ 。Kenneth J. Harris等 (2011) 以 205 名汽车公司员工作为样本, 证明领导-成员关系 (LMX) 作为组织中领导支持的力量促进员工嵌入度 的重要变量, 处于高LMX关系下的员工拥有许多的资 源, 这些资源可以补充员工的资源, 使员工感觉自己 融入其中 ${ }^{[9]}$ 。Thoms W. H. NG等（2013）也提出企业 中主管的嵌入感与员工自身的嵌入感是直接相关的, 会影响员工对企业的情感、依恋和行为, 其次组织信 任在主管嵌入与员工嵌入之间充当中介作用 ${ }^{[10]}$ 。从与 员工相关的领导同事关系出发, 为员工嵌入提供了一 个新的解释机制。

\section{2. 结果变量研究综述}

员工嵌入的结果变量研究前期主要是集中于员工 离职问题分析。Mitchell和Lee (2001) 首次提出员工 嵌入这个概念, 并用展开模型和工作嵌入性整合去预 测员工非自愿离职行为, 提出通过嵌入来增加依恋和 或通过理解展开模型的原则来减少人员流动 (Mitchell, T. R., and Lee, T. W, 2001)。Tanova 和 Holtom (2008) 也证明了在离职因素中除了态度因素之外, 还受工作 嵌入因素的影响, 即使在控制了其他变量后, 工作嵌 入仍然是影响员工嵌入的重要因素 $[11]$ 。徐茜与张体勤 （2017）探讨工作嵌入与员工流动倾向之间的关系, 通过 402 名样本调查发现, 工作嵌入内的匹配和损失显 著负向影响员工流动倾向 ${ }^{[2]}$ 。

离职研究是在工作嵌入中是研究比较成熟的领 域, 有其他学者把员工嵌入的研究转向其他员工行为 领域。如研究工作嵌入对于员工创新行为的影响。有 学者发现工作嵌入性与员工创新行为存在显著的正向 关系, 即使是控制了人口统计学项目、工作态度和工 作嵌入相关的变量, 依旧是正向关系。高度嵌入的员 工在充满安全感的环境中, 更有可能会产生创意, 并 积极进行创新行为, 以获得更多升职加薪的机会。员 工嵌入不仅仅会影响员工的创新行为, 还会影响员工 在组织中的建言。Alice 等 (2019) 研究工作嵌入下的 
员工是否会主动为组织未来的改进提供建议, 通过对于 华南地区的管理人员和主管的数据调查, 发现工作嵌入 性与建言之间有正向关系, 并且对于组织的影响也是呈 正相关, 工作嵌入性与组织之间的关系在员工的互动公 平感较低的时候更为显著 ${ }^{[13]}$ 。还有更多创新的结果变量, 例如员工建立社会资本的行为。当前员工嵌入的结果变 量主要还是员工行为, 但是研究领域在离职意愿之外的 其他创新的变量, 并且呈现继续扩大的趋势。

\section{3. 其他拓展研究方向}

有些学者研究不同领导风格对于员工行为的影响, 把员工嵌入作为调节, 如我国学者周宇 (2018) 研究包 容型领导与员工敬业度之间的关系, 把员工嵌入作为调 节加入到模型中, 拓展了工作嵌入研究方向 ${ }^{[14]}$ 。Hom P W 等 (2009) 关注组织与成员关系视角下, 把员工嵌入纳 入到社会交换对于就业的影响研究模型中, 发现工作嵌 入是相互投资和过度投资对于离职倾向和离职承诺的重 要中介。建立组织理论和领导理论的下员工嵌入解释机 制, 扩充工作嵌入解释机制[15]。有些学者把家庭方面的 研究也纳入到工作嵌入研究体系中来, 想要研究工作嵌 入性在员工对待家庭问题对工作绩效所产生的影响。 Karatepe等 (2013) 在2013年发布此类文章, 通过调查酒 店员工样本, 发现情绪耗竭作为工作负担较重、工作-家 庭冲突和家庭工作冲突对工作嵌入性和工作嵌入影响的 中介, 工作负担重的员工, 无法在工作和家庭角色之间 建立良好的平衡, 也就没办法更好的嵌入到工作中 ${ }^{[16]}$ 。 在对于工作压力大的行业提供一个好的启示。

以往研究不光关注于员工嵌入积极的部分, 有些学 者着重研究消极的方面的影响, 但是该类研究目前较少。 James B. Avey（2015）主要是探讨工作嵌入, 特别是在 滥用监督的背景下, 如何实际的导致消极的工作结果, 特别是增加工作挫折和工作离职。在员工嵌入到组织中, 相当于被 “卡住” 时。会正向调节员工的消极行为, 会 使情况变得更加糟糕 ${ }^{[17]}$ 。Shelly Marasi等 (2016) 通过353 个护士数据样本, 发现工作嵌入显著的调节了组织的信 任和工作偏差关系, 在经历在低的组织信任和高工作嵌 入比经历低组织嵌入和低工作嵌入的参与者有很多的工 作偏差 ${ }^{[18]}$ 。该类研究证明工作嵌入对于在消极环境下的 员工行为的影响很可能也是消极的。

区分不同文化特征下的员工嵌入情况也是目前研究 的一个重要方向。关注跨文化的研究比较少, 有些学者 考察个人主义国家和集体主义国家的人员流动, 研究工 作嵌入模型的跨文化概括性（Mitchell\&Lee,2001）。Gong $\mathrm{Y}$ 等 (2011) 研究中国人说方言与对一个组织的认同过程 有关, 反过来又影响他们留在组织中, 探讨中国文化差 异下的嵌入过程 ${ }^{[19]}$ 。我国学者杨春江等 (2013) 也在2013 年进行过员工嵌入的本土化研究, 通过对中国人的深度 访谈和分析探索中国背景下的员工嵌入理论 ${ }^{[20]}$ 。该学者 在2019年进行有关中国背景下的员工嵌入量表开发, 更 近一步推进了工作嵌入度在中国的发展。不同文化下理
论的发展是现在研究所欠缺的, 也是以后需要努力的 方向。

\section{5. 结论与研究展望}

从Web of science和CNKI资料库搜索工作嵌入近 30 多年文献发现，工作嵌入的文献最早是1983年，国 内外文献从2008年之后有较快的增长。2013年是国内 文献的爆发期, 除了在期刊文章数量的增长之外, 国 内硕博士论文中关于员工嵌入的研究也逐渐的增多。 从研究趋势上看外文文献研究前期集中于工作嵌入与 离职关系的分析, 国外工作嵌入研究后期呈职业分化 趋势, 像是对于特定职业的工作嵌入对于员工行为研 究的影响增多, 如研究护士行业、酒店行业员工的员 工嵌入程度对于行为的研究。国内研究集中于并开始 积极发展员工嵌入与其他员工行为如满意度、离职倾 向和工作绩效等行为之间的关系研究, 有些学者从工 作嵌入研究转向组织层面嵌入研究。后期员工嵌入研 究呈现扩散趋势, 逐渐与其他已经成熟的理论结合去 预测员工行为, 另外有学者开始探索不同文化下的工 作嵌入的量表开发和特殊背景下的嵌入的影响因素。 员工嵌入前期主要作为前因变量为研究重点, 国内外 后期发展中积极探索员工嵌入作为其他影响因素对于 其他变量的影响, 产生中介作用的文献比较多, 在员 工嵌入作为中介变量的研究领域比较热门。但是充当 调节变量比较少。

基于本篇综述提出以下三点展望，（1）以后的研 究方向可以更加的细分, 探索不同职业特征下的工作 嵌入的不同影响, 如服务行业需要更多的情绪消耗, 情绪消耗是否会因为工作嵌入影响员工的行为。（2） 更多的去探索员工嵌入充当调节的情况，员工嵌入的 前因和结果变量很多的被研究比较成熟, 可以探索在 其他理论背景才员工嵌入理论充当的调节角色。（3） 探索文化背景下的工作嵌入的影响因素，如在中国关 系型社会中, 是否能真正的通过某些因素提高员工的 嵌入度。或者其他文化背景下的工作嵌入的不同表现， 让工作嵌入理论与文化结合起来。

\section{REFERENCES}

[1] Yang ChunJiang, Ma QinHai, Zeng XianFeng (2010) Job Embeddedness:A New Perspective to Predict Voluntary Turnover .Nankai Business Review, 13: 105-118.

[2] Zhang, M, D.D. Fried ,R.W. Griffeth (2012) A review of job embeddedness: Conceptual, measurement issues, and directions for future research . Human Resource Management Review, 22: 220-231.

[3] William Lee, T., T.C. Burch and T.R. Mitchell (2014) The Story of Why We Stay: A Review of Job 
[12] Xu Qian and Zhang TiQin ( 2017 ) Job

Embeddedness .Annual Review of Organizational Psychology and Organizational Behavior,1: 199-216.

[4] Mitchell, T. R., and Lee, T. W. (2001) The unfolding model of voluntary turnover and job embeddedness: foundations for a comprehensive theory of attachment .Research in Organizational Behavior,23: 189-246.

[5] Crossley, C. D., Bennett, R. J., Jex, S. M., and Burnfield, J. L. (2007)Development of a global measure of job embeddedness and integration into a traditional model of voluntary turnover. Appl. Psychol,92 : 1031-1042.

[6] Clinton, M., Knight, T., and Guest, D. E. (2012) Job Embeddedness: A new attitudinal measure .International Journal of Selection and Assessment,20 : 111-117.

[7] Yang ChunJiang,Liu Dan,Mao ChengCheng (2019) Job embeddedness in China: An analysis of concept, structure and scale,Journal of Industrial Engineering and Engineering Management,33 : 122-133.

[8] Singh, B., M.A. Shaffer, T.T. (2018) Selvarajan, Antecedents of organizational and community embeddedness: The roles of support, psychological safety, and need to belong,Journal of Organizational Behavior, 39: 339-354.

[9] Harris, K.J., A.R. Wheeler and K.M. Kacmar (2011) The mediating role of organizational job embeddedness in the LMX-outcomes relationships. The Leadership Quarterly, 22 : 271-281.

[10] Ng, T.W.H. , D.C. Feldman (2013) Changes in Perceived Supervisor Embeddedness: Effects on Employees' Embeddedness, Organizational Trust, and Voice Behavior. Personnel Psychology, 66 : 645-685.

[11] Tanova, C., Holtom, B. ( 2008 ) Using Job Embeddedness Factors to Explain Voluntary Turnover in 4 European Countries. International Journal of Human Resource Management, $9: 1553-1568$. embeddedness and staff's flow intention:Work values as a moderator variable,Journal of Industrial Engineering and Engineering Management,31: 19-28.

[13] Tan A J M , Loi R, Lam L W, et al. (2019) Do embedded employees voice more? Personnel Review, 48 : 824-838.

[14] Zhou Yu,Fang ZhiCheng and Mi EnGuang,Inclusive Leadership (2018) Psychological Capital and Employee Engagement_-Job Embeddedness as a Moderator,Journal of Technical Economics \& Management,11 : 54-59.

[15] Hom P W,Tsui A S , Wu J B (2009) Explaining Employment Relationships With Social Exchange and Job Embeddedness, Journal of Applied Psychology,94 : 277-297.

[16] Karatepe, O.M. (2003) The effects of work overload and work-family conflict on job embeddedness and job performance, International Journal of Contemporary Hospitality Management,25 : 614-634.

[17] Avey, J.B., K. Wu , E. Holley (2015) The Influence of Abusive Supervision and Job Embeddedness on Citizenship and Deviance,Journal of Business Ethics, 129 : 21-731.

[18] Shelly Marasi, Susie S. Cox, Rebecca J Bennett (2016) "Job embeddedness: is it always a good thing?", Journal of Managerial Psychology, 31 : 141-153.

[19] Gong Y, Chow H S and Ahlstrom D (2011) Cultural diversity in China: Dialect, job embeddedness, and turnover, Asia Pacific Journal of Management, 28 : 221-238.

[20] Yang ChunJiang,Liu Wei and Luo Bing (2013) What should employees think before leaving:job embeddedness in China,Science-Technology and Management,1 : 29-33. 